\title{
Sperm binding to the pig zona pellucida and inhibition of binding by solubilized components of the zona pellucida
}

\author{
Trish Berger*, Arlene Davis*, N. J. Wardrip $\dagger$ and Jerry L. Hedrick $\dagger$ \\ Departments of *Animal Science and $\dagger$ Biochemistry and Biophysics, University of California, Davis, \\ California 95616, USA
}

\begin{abstract}
Summary. An assay to determine the binding of pig spermatozoa to the zona pellucida (ZP) of pig oocytes was developed using conditions compatible with in-vitro fertilization of pig eggs and with pig sperm penetration of zona-free hamster ova. These conditions were used to define which of the pig oocyte ZP components were involved in sperm binding by a competitive inhibition approach. Assay variables that were optimized included: the method of sperm preparation; sperm preincubation time; sperm-oocyte coincubation time, sperm concentration and temperature; and methods for the separation of free from oocyte-bound spermatozoa. Inclusion of solubilized ZP in the sperm preincubation medium inhibited sperm binding approximately $50 \%$. Both the $55 \mathrm{~K}$ and $90 \mathrm{~K}$ components inhibited sperm binding although the $55 \mathrm{~K}$ component was more effective. The two polypeptides derived from chemical deglycosylation of the $55 \mathrm{~K}$ families did not inhibit sperm binding. Of several monoclonal antibodies to the ZP components tested, only one directed against the $55 \mathrm{~K}_{\alpha}$ glycoprotein family inhibited sperm binding. Sperm binding to pig oocyte ZP is therefore dependent on the carbohydrate moiety of the glycoproteins and appears to involve more than a single ZP glycoprotein.
\end{abstract}

Keywords: zona pellucida; sperm binding; fertilization; pig

\section{Introduction}

Binding of spermatozoa to the zona pellucida (ZP) is an initial step in the fertilization process. Uncapacitated pig spermatozoa can bind to the ZP in vitro (Peterson et al., 1984). Spermatozoa continue to bind to the pig ZP after in-vivo fertilization (Hunter, 1974). We do not yet know whether inefficient sperm-ZP binding is a significant problem in fertilization failure or whether variation in sperm-ZP binding contributes to quantitative differences in fertility among fertile males. A zona-free hamster ova bioassay for pig spermatozoa which examines steps in the fertilization process after penetration of the ZP has been developed (Berger \& Horton, 1988). Although a successful technique for in-vitro fertilization in the pig has been developed (Cheng, 1985), large numbers of fertile pig ova are not readily available. The ZP composition of oocytes is very similar to that of ova (Hedrick et al., 1987) and oocytes are readily available. Hence, we chose to develop a sperm-ZP binding assay using oocytes that was compatible with sperm preparation for the zona-free hamster ova bioassay.

The pig ZP consists of three glycosylated polypeptide chains which are referred to by a nomenclature based on apparent molecular weight as the $55 \mathrm{~K}_{a}, 55 \mathrm{~K}_{\beta}$, and $90 \mathrm{~K}$ families (Hedrick \& Wardrip, 1986, 1987). Under conditions in which disulphide bonds are reduced, a $25 \mathrm{~K}$ component and a $65 \mathrm{~K}$ component are derived from a portion of the $90 \mathrm{~K}$ family. We also wished to examine which of the pig ZP glycoprotein families were involved in sperm binding. 


\section{Materials and Methods}

Pig ZP were prepared as previously described (Hedrick \& Wardrip, 1986). Briefly, pig ovaries were obtained from the slaughter house and stored frozen until use. The follicles were ruptured using a ganged razor blade apparatus. Zona pellucida-intact oocytes were separated from debris by multiple sievings through nylon screens of different mesh sizes. These oocytes were stored at $4^{\circ} \mathrm{C}$ in PBS with azide for up to 10 days before use. On the day of use, oocytes were rinsed through 4 1-ml volumes of Tris-buffered medium (113.1 mM- NaCl, $3 \mathrm{~mm}-\mathrm{KCl}, 20 \mathrm{~mm}-\mathrm{Tris}$, $11 \mathrm{~mm}$-glucose, $5 \mathrm{~mm}-$ sodium pyruvate, and $50 \mu \mathrm{g}$ gentamycin sulphate $/ \mathrm{ml}$; Berger \& Horton, 1988) containing $40 \mathrm{mM}-\mathrm{CaCl}_{2}$ and $5 \mathrm{mg}$ bovine serum albumin/ml (BSA; $\$$ A7906, Sigma, St Louis, MO, USA). Any oocytes with a damaged ZP were removed.

Ejaculated spermatozoa from the sperm-rich fraction were collected and processed as previously described (Berger \& Horton, 1988) using Tris-buffered medium containing $40 \mathrm{~mm}-\mathrm{CaCl}_{2}$ and $5 \mathrm{mg} \mathrm{BSA} / \mathrm{ml}$. Processed spermatozoa were preincubated in $2 \mathrm{ml}$ volumes $\left(2 \times 10^{6}\right.$ spermatozoa $/ \mathrm{ml}$ at $39^{\circ} \mathrm{C}$ for $1 \mathrm{~h}$ in $5 \% \mathrm{CO}_{2}$ in air. The sperm suspension was then diluted to $2 \times 10^{\mathrm{s}}$ spermatozoa/ml and $100 \mu \mathrm{l}$ were used to inseminate a $100-\mu \mathrm{l}$ volume of medium containing 100 oocytes. Unless otherwise stated, gametes were coincubated for $3 \mathrm{~h}$ at $39^{\circ} \mathrm{C}$ in $5 \% \mathrm{CO}_{2}$ in air. Oocytes were separated from the unbound spermatozoa using a modification of the stop-fix procedure described by Saling et al. (1978). A dextran gradient was prepared in a $1.0-\mathrm{ml}$ polystyrene centrifuge tube (Fisher) by layering $0.35 \mathrm{ml}$ of $1.8 \%$ dextran (average molecular weight 170000 ) in Tris-buffered medium containing BSA above $0.35 \mathrm{ml}$ of $2.25 \%$ dextran in the same medium. The oocytes in $100 \mu \mathrm{l}$ medium were then layered on top and centrifuged for $3 \mathrm{~min}$ at $160 \mathrm{~g}$. All but $\sim 100 \mu \mathrm{l}$ was aspirated and the oocytes resuspended in $1 \mathrm{ml} 0.625 \%$ glutaraldehyde. They were allowed to settle, the first glutaraldehyde solution aspirated and an additional $1 \mathrm{ml}$ glutaraldehyde solution was used to transfer oocytes to a dish. A $1-\mathrm{ml}$ syringe fitted with a 26-gauge needle and filled with a mixture of vaseline:paraffin wax (8:1 w/w) was used to form a ribbon along each long edge of a cleaned microscope slide. The oocytes were placed between the 2 ribbons and slightly compressed with a coverslip. Excess fluid was removed and the coverslip sealed with mounting medium. Spermatozoa bound to hemispheres of each of 30 oocytes were counted using differential interference contrast optics at $\times 400$ magnification.

When indicated, heat-solubilized pig ZP or isolated ZP components were added to the spermatozoa during preincubation. ZP and ZP components were isolated by preparative two-dimensional polyacrylamide gel electrophoresis as previously described (Hedrick \& Wardrip, 1986, 1987). Sodium dodecyl sulphate (SDS) was extracted from isolated components or from BSA previously mixed with SDS using Extracti-Gel D (Pierce Chemical Co., Rockford, IL, USA).

A total of 12 monoclonal antibodies to zona pellucida components were evaluated for their effect on sperm -oocyte ZP binding (Betancourt et al., 1984, 1985; Hedrick \& Wardrip, 1987). Oocytes were preincubated at $39^{\circ} \mathrm{C}$ for $45 \mathrm{~min}$ with each monoclonal antibody at a 1:100 dilution of the ascites fluid, rinsed in three 1.0 ml volumes of medium to remove free antibody, and coincubated with spermatozoa.

To develop the assay conditions, 3-5 ejaculates from a minimum of 3 boars were used. A minimum of 1 ejaculate from each of 3 boars was used in subsequent experiments. Analyses of variance and regression were performed on mean numbers of spermatozoa bound per oocyte using the BMDP statistical package (BMDP Statistical Software, Inc, Los Angeles, CA, USA). Differences among means were evaluated with Student-Newman-Keul's sequential range test. Percentage motilities were analysed both before and after transformation to the arcsin square root and statistical significance resulting from the transformation is reported.

\section{Results}

\section{Optimization of the ZP-binding assay conditions}

Varying the sperm preincubation time from 1 to $4 \mathrm{~h}$ and the sperm-oocyte coincubation time from 1 to $3 \mathrm{~h}$ had little effect on the number of spermatozoa bound per oocyte $(P=0.33)$. However, very short incubation times $(0 \mathrm{~h}$ of sperm preincubation followed by $1 \mathrm{~h}$ of sperm-oocyte coincubation) appeared insufficient for maximum sperm binding $(P=0.15)$. Increasing the sperm preincubation time from $3 \mathrm{~h}$ to 5 or $7 \mathrm{~h}$ decreased the number of spermatozoa bound $(24,16$ and 7 spermatozoa/oocyte, respectively; $P<0.05$ ). As expected, the number of spermatozoa bound per oocyte increased with increasing sperm concentration $\left(15,35,42\right.$ and 130 for $4 \times 10^{4}, 1 \times 10^{5}$, $4 \times 10^{5}$ and $1 \times 10^{6}$ spermatozoa $\left./ \mathrm{ml} ; P<0.005\right)$. Sperm-oocyte coincubation at $39^{\circ} \mathrm{C}$ compared to room temperature increased the number of spermatozoa bound per oocyte regardless of whether the spermatozoa were preincubated for $1 \mathrm{~h}$ at $39^{\circ} \mathrm{C}(60$ vs 22 spermatozoa/oocyte, s.e.m. $=5$; $P<0.01$ ) or not preincubated (53 vs 32 spermatozoa/oocyte, s.e.m. $=5, P<0.05$ ). Sperm motility ranged from 86 to $94 \%$ at the end of the coincubation periods in these experiments and did not differ among treatments. Preincubation and coincubation at $2-10^{\circ} \mathrm{C}$ reduced binding even further 
(1 spermatozoon/oocyte vs 54 and 34 spermatozoa/oocyte for coincubation at $39^{\circ} \mathrm{C}$ and room temperature, respectively; $P<0.05$ ). In this case, however, motility was reduced to $22 \%$ after incubation at $2-10^{\circ} \mathrm{C}$.

Washing spermatozoa by dilution and centrifugation compared to washing on the Percoll gradient before preincubation and/or coincubation of gametes reduced the number of bound spermatozoa by $30 \%(P<0 \cdot 05)$. Coincubation of gametes in the absence of BSA greatly reduced sperm binding regardless of sperm washing procedures $(3,3,35$ and 57 spermatozoa/oocyte for samples washed by dilution or on a Percoll gradient and coincubated without BSA and for samples washed by dilution or on a Percoll gradient and coincubated with BSA; $P<0.01$ ). However, motility was greatly reduced after incubation in the absence of BSA (27\% and $15 \%$ for spermatozoa washed on a Percoll gradient and spermatozoa washed by dilution $v s 87 \%$ and $73 \%$ for spermatozoa prepared by the same procedures and incubated with BSA). Preincubation and coincubation with $5 \mathrm{mg}$ polyvinyl alcohol/ml (PVA, average mol. wt 10 000; Sigma, St Louis, MO, USA) also supported binding of spermatozoa to the ZP (74 and 65 spermatozoa/oocyte for BSA and PVA, respectively).

Separation of oocytes and bound spermatozoa from unbound spermatozoa on a dextran gradient was equivalent to separation by centrifugation of oocytes through $5 \%$ Percoll ( 85 and 90 spermatozoa/oocyte, s.e.m. $=9$ ). However, the number of bound spermatozoa was greatly reduced when oocytes were layered on $5 \%$ Ficoll-Paque (Pharmacia, Piscataway, NJ, USA; 42 spermatozoa/oocyte, $P<0.05$ ). Oocyte recovery was also reduced to $<50 \%$ on the Ficoll-Paque. The length of centrifugation on the dextran gradient did not affect the number of spermatozoa bound (56, 62 and 54 spermatozoa/oocyte for 2, 3 and $4 \mathrm{~min}$; s.e.m. $=5$ ). The number of bound spermatozoa did not differ when oocytes were washed free of unbound spermatozoa in 3 drops of medium using a micropipette (as described by Peterson et al., 1980) compared to the dextran gradient ( 75 and 82 spermatozoa/oocyte). The dextran gradient is therefore comparable to washing through 3 drops in measuring bound spermatozoa, not attached spermatozoa (Hartmann et al., 1972). However, since the micropipette method is pipette-dependent, i.e. the shear force is a function of the pipette bore, the dextran gradient centrifugation method is ultimately more reproducible.

The variability of sperm binding in 3-6 ejaculates from each of 6 previously fertile boars is shown in Fig. 1. This variability was not due to age of oocytes used nor was it primarily due to dayto-day variability in assay conditions since variability among ejaculates from different boars analysed on the same day was as great as the day-to-day variability in ejaculates from the same boar.

\section{Effect of solubilized ZP and isolated ZP components on sperm-oocyte ZP binding}

Preincubation of spermatozoa for $1 \mathrm{~h}$ with 25,50 or $100 \mu \mathrm{g}$ solubilized $\mathrm{ZP} / \mathrm{ml}$ significantly reduced sperm-oocyte $\mathrm{ZP}$ binding compared to preincubation without solubilized $\mathrm{ZP}$ or with $15 \mu \mathrm{g}$ $\mathrm{ZP} / \mathrm{ml}(35,32$ and 28 vs 65 and 66 spermatozoa/oocyte, s.e.m. $=6, P<0.001)$. Regression analysis indicated that spermatozoa from the 6 boars examined differed in their dose response $(P<0.05)$.

Sperm binding was reduced from 52 spermatozoa/oocyte (mean of 6 ejaculates) to 34 and 26 spermatozoa/oocyte when spermatozoa were preincubated with disulphide bond-reduced or nonreduced $55 \mathrm{~K}$ components at $30 \mu \mathrm{g} / \mathrm{ml}$ (s.e.m. $=4 ; P<0.001$ ). Preincubation with $30 \mu \mathrm{g}$ of SDStreated BSA $/ \mathrm{ml}$ (as described in 'Materials and Methods') did not affect subsequent binding (58 spermatozoa/oocyte). Preincubation of spermatozoa from 6 ejaculates with $5 \mu \mathrm{g} 25 \mathrm{~K}$ components/ $\mathrm{ml}$ or $10 \mu \mathrm{g} 65 \mathrm{~K}$ components $/ \mathrm{ml}$ did not inhibit sperm binding ( 75 and 62 vs 70 spermatozoa/oocyte in the absence of $\mathrm{ZP}$ components, s.e.m. =6). However, preincubation with $10 \mu \mathrm{g} / \mathrm{ml}$ of the $90 \mathrm{~K}$ family had a slight effect on binding (53 spermatozoa/oocyte, $P=0.06$ in the above experiment and $64 v s 48$ spermatozoa/oocyte in the absence and presence of $10 \mu \mathrm{g} / \mathrm{ml}$ of $90 \mathrm{~K}$ family, s.e.m. $=4$, 


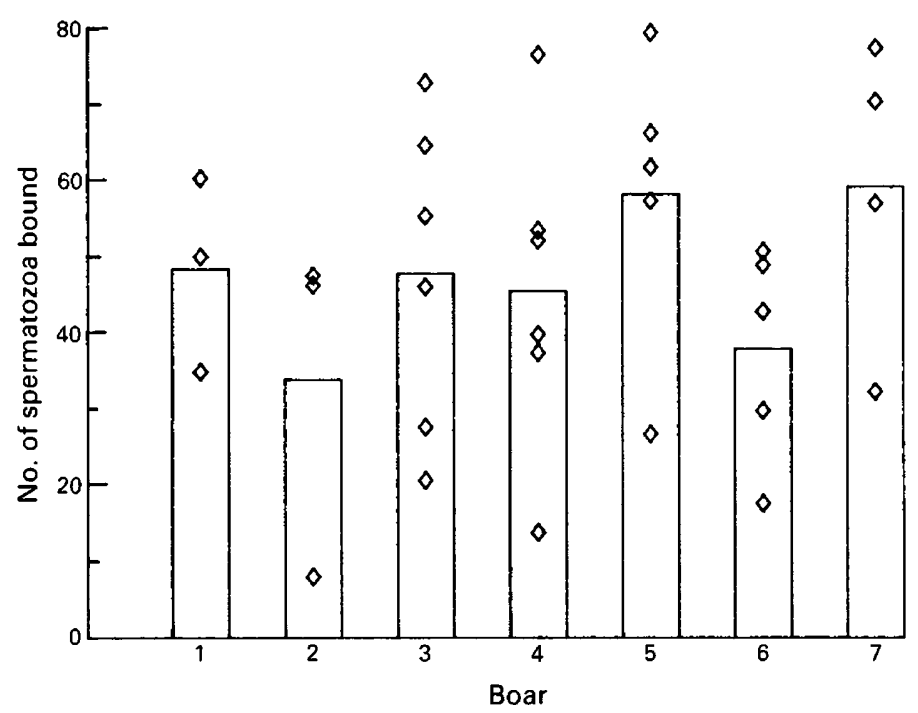

Fig. 1. Variability in sperm-oocyte ZP binding among ejaculates from fertile boars. Spermatozoa were washed on a Percoll gradient, preincubated for $1 \mathrm{~h}$ at $39^{\circ} \mathrm{C}$, and coincubated with $\mathrm{ZP}$-intact oocytes for $3 \mathrm{~h}$. Each point represents the mean number of spermatozoa/oocyte for spermatozoa from one ejaculate. Each bar represents the mean number of spermatozoa/oocyte for spermatozoa from 3-6 ejaculates from an individual boar.

Table 1. Effect of ZP molecules on sperm-ZP binding

\begin{tabular}{lcc}
\hline & \multicolumn{2}{c}{ Effect on sperm-ZP binding } \\
\cline { 2 - 3 } ZP molecule & $\begin{array}{c}\text { \% of control } \\
\text { sperm-ZP binding }\end{array}$ & Significance \\
\hline $25-50 \mu \mathrm{g} \mathrm{ZP} / \mathrm{ml}$ & $49-54$ & $P<0.001$ \\
$30 \mu \mathrm{g} 55 \mathrm{~K}($ reduced $) / \mathrm{ml}$ & 65 & $P<0.001$ \\
$30 \mu \mathrm{g} 55 \mathrm{~K}($ non-reduced $) / \mathrm{ml}$ & 50 & $P<0.001$ \\
$10 \mu \mathrm{g} 90 \mathrm{~K} / \mathrm{ml}$ & $75-76$ & $P<0.05$ \\
$10 \mu \mathrm{g} 65 \mathrm{~K} / \mathrm{ml}$ & 89 & $\mathrm{NS}$ \\
$5 \mu \mathrm{g} 25 \mathrm{~K} / \mathrm{ml}$ & 107 & $\mathrm{NS}$ \\
$15 \mu \mathrm{g} \mathrm{ZP} \mathrm{polypeptide}$ & & $\mathrm{NS}$ \\
$\left(M_{\mathrm{r}} 37000\right) / \mathrm{ml}$ & 111 & $\mathrm{NS}$ \\
$15 \mu \mathrm{g} \mathrm{ZP} \mathrm{polypeptide}$ & & \\
$\left(M_{\mathrm{r}} 40000\right) / \mathrm{ml}$ & 100 & \\
\hline
\end{tabular}

NS, not significant.

$P<0.05$, in a separate experiment involving spermatozoa from 10 ejaculates). The concentrations of individual ZP components used were in approximate proportion to their composition in the ZP.

Preincubation of spermatozoa with $15 \mu \mathrm{g} / \mathrm{ml}$ of the $M_{\mathrm{r}} 37000$ and 40000 polypeptides prepared by chemical deglycosylation of the $55 \mathrm{~K}$ glycoproteins did not affect binding (47, 52 and 47 spermatozoa/oocyte for control, $M_{\mathrm{r}} 37000$ and $M_{\mathrm{r}} 40000$ vs 31 spermatozoa/oocyte after preincubation with $30 \mu \mathrm{g} 55 \mathrm{~K}$ components $/ \mathrm{ml}$ for spermatozoa from 10 ejaculates, s.e.m. $=4, P=0.02$ ). 
In this experiment, motility ranged from 90 to $92 \%$ at insemination and from 81 to $87 \%$ after coincubation and did not differ among treatments.

\section{Effect of glucosaminidase on sperm-oocyte ZP binding}

Preincubation of oocytes with $\beta$ - $N$-acetylglucosaminidase (Sigma, St Louis, MO, USA; \#A2264 from jack bean) eliminated the ability of spermatozoa to bind to the ZP ( 8 spermatozoa/oocyte for oocytes preincubated for $2 \mathrm{~h}$ with 6 IU $\beta$ - $N$-acetylglucosaminidase $/ \mathrm{ml}$ and rinsed in 3 drops of medium before insemination vs 49 spermatozoa/oocyte for oocytes preincubated in medium alone, mean of 4 ejaculates, s.e.m. $=6 ; P<0.005$ ). Preincubation of oocytes with 6 IU $\beta$-galactosidase/ $\mathrm{ml}$ (Sigma; $¥ 6512$ from $E$. coli) did not affect binding ( 59 spermatozoa/oocyte).

\section{Effect of monoclonal antibodies to ZP on sperm-oocyte ZP binding}

One monoclonal antibody to the peptide moiety of the $55 \mathrm{~K}_{\mathrm{a}}$ glycoprotein significantly inhibited sperm-oocyte ZP binding (40 vs 80 spermatozoa/oocyte in the absence of monoclonal antibodies in the first experiment utilizing an ejaculate from each of 3 boars, s.e.m. $=8$; and 31 vs 56 spermatozoa/oocyte in the second trial using one ejaculate from each of 5 boars, s.e.m. $=7$ ). The other monoclonal antibodies ( 2 to the peptide moiety of the $55 \mathrm{~K}_{\beta}$ glycoprotein, 1 to the peptide moiety of the $25 \mathrm{~K}$ component derived from the $90 \mathrm{~K}$ family, 2 to the peptide moiety of the $65 \mathrm{~K}$ component derived from the $90 \mathrm{~K}$ family, 2 which react with carbohydrate of all families, 3 which are conformation-dependent and react only with intact or heat-solubilized ZP but not with SDSdissociated ZP, and an additional monoclonal antibody to the peptide moiety of the $55 \mathrm{~K}_{\alpha}$ glycoprotein) did not significantly inhibit sperm binding. The monoclonal antibody to the $55 \mathrm{~K}_{\alpha}$ glycoprotein had a dose-response effect on sperm binding (Fig. 2). Dose-response studies (extending from a 1:5 to 1:100 dilutions of the monoclonal antibodies in ascites fluid) indicated no effect compared to control ascites fluid dilutions for two additional monoclonal antibodies, one specific to the peptide moiety of the $55 \mathrm{~K}_{\beta}$ glycoprotein and one specific to the carbohydrate moiety of all components.

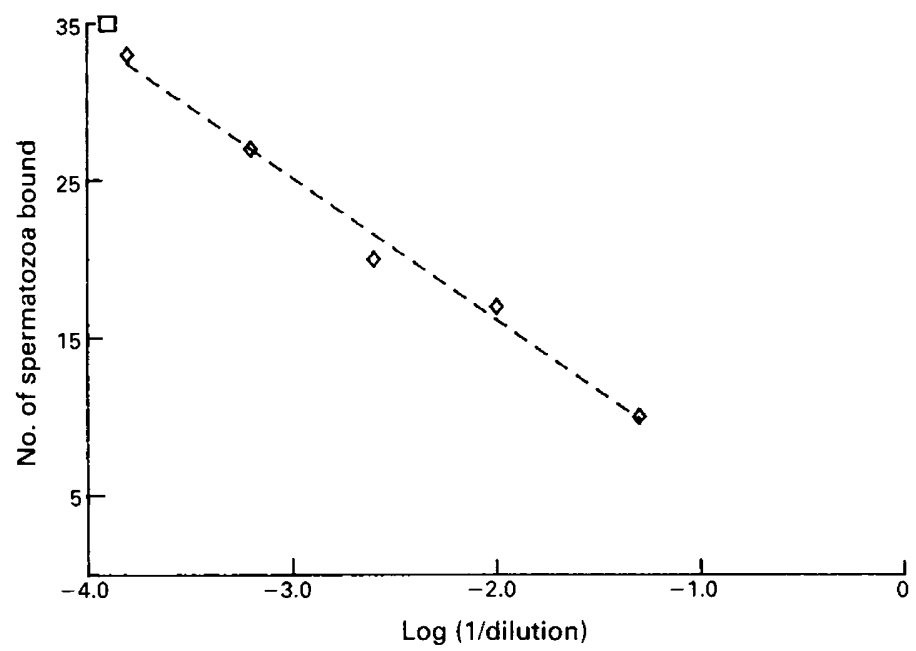

Fig. 2. Sperm-oocyte ZP binding after preincubation of oocytes with various concentrations of a monoclonal antibody to $55 \mathrm{~K}_{\alpha}$. Oocytes were preincubated for $45 \mathrm{~min}$ with an $\mathrm{IgG}_{1}$ monoclonal antibody (123-7) and rinsed 3 times before coincubation with spermatozoa. $\diamond$, Mean no. of spermatozoa/oocyte for oocytes preincubated with the indicated concentration of antibody; $\square$, mean no. of spermatozoa/oocyte for oocytes preincubated with medium alone. 


\section{Discussion}

Peterson et al. (1984) reported binding by washed uncapacitated pig spermatozoa. Since the gamete interaction occurred in the presence of BSA at room temperature, BSA may have promoted partial capacitation in the previous study. In this study, no substantial binding occurred when gametes were incubated in the absence of BSA or polyvinyl alcohol but this was probably due to the decrease in motility. Polyvinyl alcohol was a satisfactory substitute for BSA in permitting binding. However, spermatozoa incubated with polyvinyl alcohol are not sufficiently capacitated to penetrate zonafree hamster ova (Berger \& Parker, 1989). Hence, our observations are in agreement with the previous conclusion that uncapacitated pig spermatozoa bind to the ZP. Seminal plasma proteins reportedly inhibit binding of pig spermatozoa to ZP (Peterson et al., 1984). Removal of such proteins probably occurs during capacitation (Brackett \& Oliphant, 1975). Removal of adsorbed seminal plasma proteins during the washing procedure on the Percoll gradient could explain why such spermatozoa bound to a greater extent than did spermatozoa washed by dilution. One might also describe spermatozoa washed on the Percoll gradient as partly capacitated since such spermatozoa will penetrate zona-free hamster ova whereas spermatozoa washed by dilution and incubated under the same conditions have a reduced ability to penetrate zona-free hamster ova (Berger \& Horton, 1988). Probably, binding of partly capacitated spermatozoa and uncapacitated spermatozoa occurs in the pig as in the hamster.

Day-to-day variability in the numbers of spermatozoa bound to the ZP has been observed in the pig and the mouse (Peterson et al., 1980; Florman et al., 1984). The day-to-day variability we observed was not due to the age of the ZP but may have been at least partly due to day-to-day fluctuations in the ejaculates.

Solubilized ZP strongly inhibited sperm-oocyte binding. The solubilized $55 \mathrm{~K}$ component also strongly inhibited sperm binding, indicating that at least one of the $55 \mathrm{~K}$ families is involved in sperm binding. Inhibition studies with monoclonal antibodies suggest that the $55 \mathrm{~K}_{\alpha}$ family is a major ZP ligand for spermatozoa. The $55 \mathrm{~K}$ component corresponds to the $58 \mathrm{~K}$ PPZA described by Sacco et al. (1984) who reported inhibition of ZP binding by spermatozoa from 2 ejaculates. However, other components of the ZP were not examined in their previous study. In this study, the $25 \mathrm{~K}$ and $65 \mathrm{~K}$ components, which under non-reducing conditions comigrate with the $90 \mathrm{~K}$ family and are derived from this family, had no effect on sperm-oocyte ZP binding. The $90 \mathrm{~K}$ component had a small but significant effect.

The 55K component of the pig ZP is composed of 2 glycoprotein families which have been termed $55 \mathrm{~K}_{\alpha}$ and $55 \mathrm{~K}_{\beta}$ (Hedrick \& Wardrip, 1987). The stoichiometric composition of the $55 \mathrm{~K}_{\alpha}$, $55 \mathrm{~K}_{\beta}$, and $90 \mathrm{~K} \mathrm{ZP}$ glycoprotein families is approximately 1:1:1. Each of the glycoprotein families possesses extreme microheterogeneity primarily due to variation in the carbohydrate moiety (Dunbar et al., 1980; Sacco et al., 1981). However, we do not yet know if the variation in oligosaccharide composition affects sperm binding. Inhibition of sperm-oocyte ZP binding by a monoclonal antibody to the $55 \mathrm{~K}_{\mathrm{a}}$ glycoprotein and lack of inhibition by other monoclonal antibodies suggests that the $55 \mathrm{~K}_{a}$ family is a major ZP ligand for spermatozoa.

The polypeptides of $M_{\mathrm{r}} 37000$ and 40000 derived from the $55 \mathrm{~K}_{\alpha}$ and $55 \mathrm{~K}_{\beta}$ families had no inhibitory activity on sperm-oocyte ZP binding. The lack of effect of the polypeptides suggests that the carbohydrate portion is essential for sperm-oocyte ZP binding in the pig; this is consistent with what has been reported for the mouse (Florman \& Wassarman, 1985). Further evidence indicating the importance of the carbohydrate portion is the observation that preincubation of the ZP-intact oocytes with $\beta$ - $N$-acetylglucosaminidase, which presumably is removing terminal $N$-acetylglucosamine residues, almost completely abolished sperm-oocyte ZP binding. Inhibition of binding by a monoclonal antibody to the peptide portion of the $55 \mathrm{~K}_{\alpha}$ family is compatible with a role for carbohydrate in the sperm binding process since binding of a relatively large $\left(M_{\mathrm{r}} 160000\right)$ antibody in the immediate vicinity of the sperm-binding site could sterically hinder sperm binding to the carbohydrate moiety. 
To understand further sperm-oocyte ZP binding in the pig, additional binding studies using isolated glycoproteins of the $55 \mathrm{~K}_{\alpha}, 55 \mathrm{~K}_{\beta}$ and $90 \mathrm{~K}$ families and their derived oligosaccharides moieties are needed.

We thank Mary Horton, Susan Dixon and D. Goldhawk for technical assistance. Research was supported in part by USDA grant 85-CRCR-1-1855.

\section{References}

Berger, T. \& Horton, M.B. (1988) Evaluation of assay conditions for the zona-free hamster ova bioassay of boar sperm fertility. Gamete Res. 19, 101-111.

Berger, T. \& Parker, K. (1989) Modification of the zonafree hamster ova bioassay of boar sperm fertility and correlation with in vivo fertility. Gamete Res. 22, (in press).

Betancourt, M., Rodriguez, E., Serrano, H., Wardrip, N. \& Hedrick, J.L. (1984) Monoclonal antibodies against the $55 \mathrm{~K}$ glycoproteins isolated from the pig zona pellucida. $J$ cell Biol. 99,395 a, abstr.

Betancourt, M., Rodriguez, H., Serrano, H., Wardrip, N. \& Hedrick, J.L. (1985) El uso de anticuerpos monoclonales para estudiar las funciones de la zona pelucida de ovulos de mamiferos. Salud Publica Med. 27, 203-212.

Brackett, B.G. \& Oliphant, G. (1975) Capacitation of rabbit spermatozoa in vitro. Biol. Reprod. 12, 260-274.

Cheng, W.T.K. (1985) Test-tube piglets: studies on fertilization of pig oocytes in vitro. J Taiwan Livest. Res. 18, 99-142.

Dunbar, B.S., Wardrip, N.J. \& Hedrick, J.L. (1980) Isolation, physiochemical properties, and macromolecular composition of zona pellucida from porcine oocytes. Biochemistry, NY2, 356-365.

Florman,H.M. \& Wassarman, P.M. (1985) O-linked oligosaccharides of mouse egg ZP3 account for its sperm receptor activity. Cell 41, 313-324.

Florman, H.M., Bechtol, K.B. \& Wassarman, P.M. (1984) Enzymatic dissection of the functions of the mouse egg's receptor for sperm. Devl Biol. 106, 243-255.

Hartmann, J.F., Gwatkin, R.B. \& Hutchison, C.F. (1972) Early contact interactions between mammalian gametes in vitro: evidence that the vitellus influences adherence between sperm and zona pellucida. Proc. natn. Acad. Sci. USA 69, 2767-2769.

Hedrick, J.L. \& Wardrip, N.J. (1986) Isolation of the zona pellucida and purification of its glycoprotein families from pig oocytes. Analyt. Biochem. 157, $63-70$.

Hedrick, J.L. \& Wardrip, N.J. (1987) On the macromolecular composition of the zona pellucida from porcine oocytes. Devl Biol. 121, 478-488.

Hedrick, J.L., Wardrip, N.J. \& Berger, T. (1987) Differences in the macromolecular composition of the zona pellucida isolated from pig oocytes, eggs, and zygotes. J. exp. Zool. 241, 257-262.

Hunter, R.H.F. (1974) Chronological and cytological details of fertilization and early embryonic development in the domestic pig, Sus scrofa. Anat. Rec. 178, $169-186$.

Peterson, R.N., Russell, L., Bundman, D. \& Freund, M. (1980) Sperm-egg interaction: evidence for boar sperm plasma membrane receptors for porcine zona pellucida. Science, NY 207, 73-74.

Peterson, R.N., Russell, L.D. \& Hunt, W.P. (1984) Evidence for specific binding of uncapacitated boar spermatozoa to porcine zonae pellucidae in vitro. $J$. exp. Zool. 231, 137-147.

Sacco, A.G., Yurewicz, E.C., Subramanian, M.G. \& DeMayo, F.J. (1981) Zona pellucida composition: species cross reactivity and contraceptive potential of antiserum to a purified pig zona antigen (PPZA). Biol. Reprod. 25, 997-1008.

Sacco, A.G., Subramanian, M.G. \& Yurewicz, E.C. (1984) Association of sperm receptor activity with a purified pig zona antigen (PPZA). J. Reprod. Immunol. 6, 89103.

Saling, P.M., Storey, B.T. \& Wolf, D.P. (1978) Calciumdependent binding of mouse epididymal spermatozoa to the zona pellucida. Devl Biol. 65, 51 5-525.

Received 10 October 1988 